\title{
Suspected endogenous endophthalmitis but proven hla associated panuveitis.
}

\author{
Shweta Dhiman* \\ Department of Ophthalmology, Delhi, India
}

\begin{abstract}
Purpose: To report a case of 33 year gentleman with foot cellulitis suspected to have endogenous endophthalmitis. On Investigations was found to be a case of HLA B27 panuveitis.
\end{abstract}

Keywords: Endophthalmitis, pupillary area, anterior capsule, chronic backache.

Accepted on April 22, 2017

\begin{abstract}
Abbreviations: RE: Right Eye; LE: Left Eye; O/E: On Examination; OD: Right Eye; OS: Left Eye; DT: Digital Tension; PL: Perception of Light; PR: Projection of Rays; S/L/E: Slit Lamp Examination; AC: Anterior Chamber; USG: Ultrasound; CXR: Chest Ray; ESR: Erythrocyte Sedimentation Rate; TLC: Total Leukocyte Count
\end{abstract}

\section{Introduction}

Uveitis is one of the major causes of blindness in the world. It involves inflammation of uveal tract. However, practically there is always some inflammation of the adjacent structures such as retina, vitreous, sclera and cornea. It can be classified according to anatomical area affected, on the basis of onset, pathologically into suppurative and non-suppurative and etiologically (DUKE ELDER'S). The major differential includes endophthalmitis. Since the evaluation involves a long set of investigations, one must be meticulous in choosing as appropriate especially in ruling out the differentials. Below we are presenting the stepwise approach we did in our case.

\section{Case Presentation}

A 33 year gentleman, engineer by occupation, presented with insidious onset painful gradual diminution of vision in his Left eye for 2 days. Patient had history of cellulitis of right foot and was on treatment since one month (broad spectrum oral antibiotics). He had no history of similar complaints before or in other eye. No complaints of pain in the infected foot on presentation On Examination: vision OD- 6/6, OS- PL+ PR accurate. Slit lamp examination of LE showed AC cells 4+ with exudates in pupillary area and $0.5 \mathrm{~mm}$ hypopyon. DT was normal, fundus was hazily seen. LE USG-B scan showed dense vitritis with few vitreous membranes. Examination of Right eye was normal except for few pigments over anterior capsule. Blood investigations showed high ESR (72) with slightly higher TLC count $(14,239)$. Chest x-ray was normal and Montoux test was negative for tuberculosis. Lower limb examination: no apparent swelling or tenderness. Considering, improved status of cellulitis and picture of right eye, the intravitreal was deferred. Investigating further came up the history of chronic backache for which he was taking anti-inflammatory drugs on and off. Following this history, aqueous tap was planned and HLA profile with $\mathrm{x}$-ray sacroiliac joint was done (Figure 1).

\section{Methods}

A 33year gentleman, presented with sudden onset painful diminution of vision of LE for 2 days. Patient was taking treatment for foot cellulitis since 1 month. No history of similar complaints before or in other eye. O/E: vision OD6/6, OS- PL+ PR accurate. LE- S/L/E showed AC cells 4+ with exudates in pupillary area and $0.5 \mathrm{~mm}$ hypopyon. DT was normal, fundus was hazy. USG: dense vitritis with few vitreous membranes.RE normal except for few pigments over anterior capsule. Investigations showed high ESR with slightly higher TLC count, negative CXR and montoux. Considering, improved status of cellulitis and picture of RE, the intravitreal was deferred. Investigating further came up history of chronic backache. Aqueous tap was sent which showed inflammatory cells and HLA profile was done and was positive.

\section{Discussion}

Uveitis is the inflammation of uveal tract which can involve retina. The common causes of uveitis are infective, allergic, toxic, traumatic, auto-immune diseases. Most of the patients are idiopathic [1,2]. While evaluating such cases one should be meticulous in treating according to underlying cause to prevent undue expenditure on exhaustive list on investigations. A tailored approach should be followed based on history and clinical examination [3].

Endogenous uveitis is caused by entrance of organism from source situated elsewhere in the body by the way of bloodstream. Secondary infection from nearby structures like keratitis, sclerits, orbital cellulitis can also lead to involvement of uvea. In such cases the treatment of underlying inciting cause by systemic or topical antibiotics along with anti-inflammatory drugs may improve the clinical picture. However, in cases of endogenous endophthalmitis the management protocol changes to vigorous intervention by giving intravitreal antibiotics with or without intravenous antibiotics depending upon vitreous tap report. Cellulitis is non-necrotizing inflammation of the skin and subcutaneous tissues usually following a breach in the skin. Most common cause is staphylococcus. Patients with severe cellulitis require parenteral therapy [4]. However milder cases are treated with oral antibiotics like Dicloxacillin, amoxicillin, or cephalexin as in our case (Figure 2). 


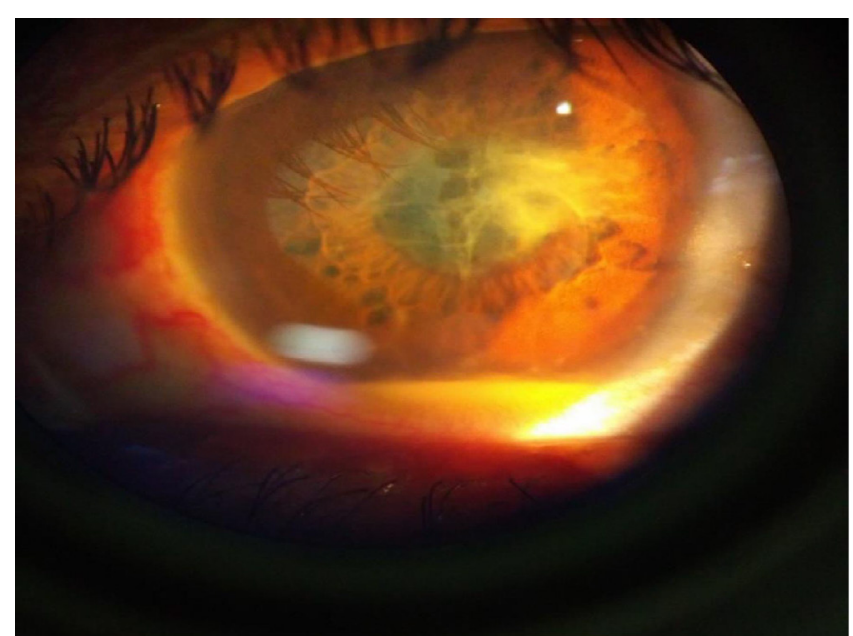

Figure 1. Showing clinical picture of left eye of the patient at presentation..

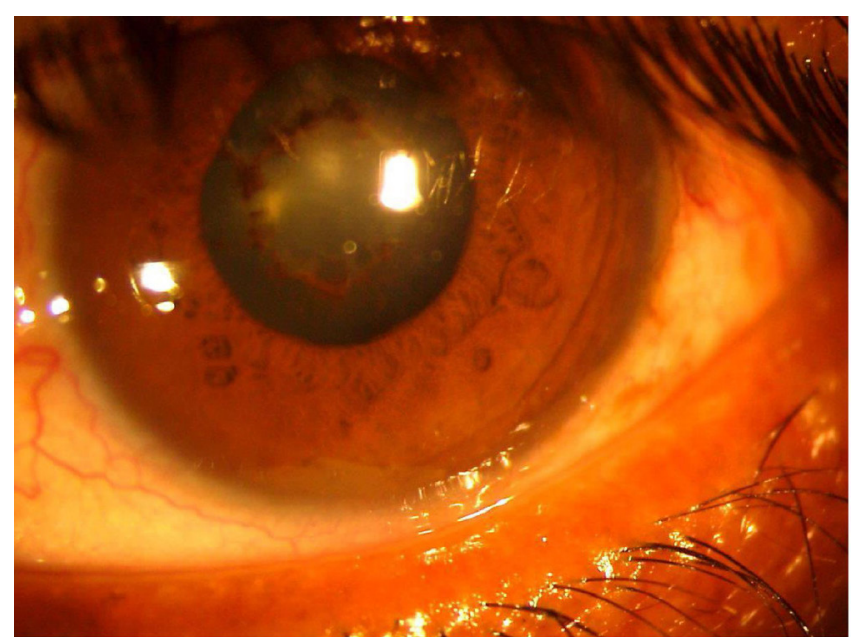

Figure 2. showing clinical picture at 2 weeks post treatment.

In uveitic patient a battery of tests is not usually required. One should concentrate on a limited number as suggested by the case. In our setup we routinely undertake investigations to rule out tuberculosis. HLA, RA, X-ray sacroiliac joint are done when history is positive. The mainstay of the treatment of uveitis is corticosteroids. Immunosuppressive agents are administered if the inflammation is not adequately controlled with steroids.

The clinical situation grossly improved in our case with oral and topical steroids. In a nutshell, the patient was treated for cellulitis with oral antibiotics, suggests the importance of meticulous history taking as a whole besides a good ophthalmological evaluation of affected and normal eye keeping all the differentials in mind as uveitis is associated with many systemic diseases (Figure 3).

\section{Results}

The patient started on 2hourly topical steroids and oral prednisolone $1 \mathrm{mg} / \mathrm{kg} /$ day for a week with gradual tapering every week. Vision after 2 weeks: OD- 6/6, OS- 6/18(P) with normal IOP and resolved pupillary membranes and vitritis.

Aqueous tap showed inflammatory cells mainly polymorphonuclear subtype. HLA test was positive for B27 and

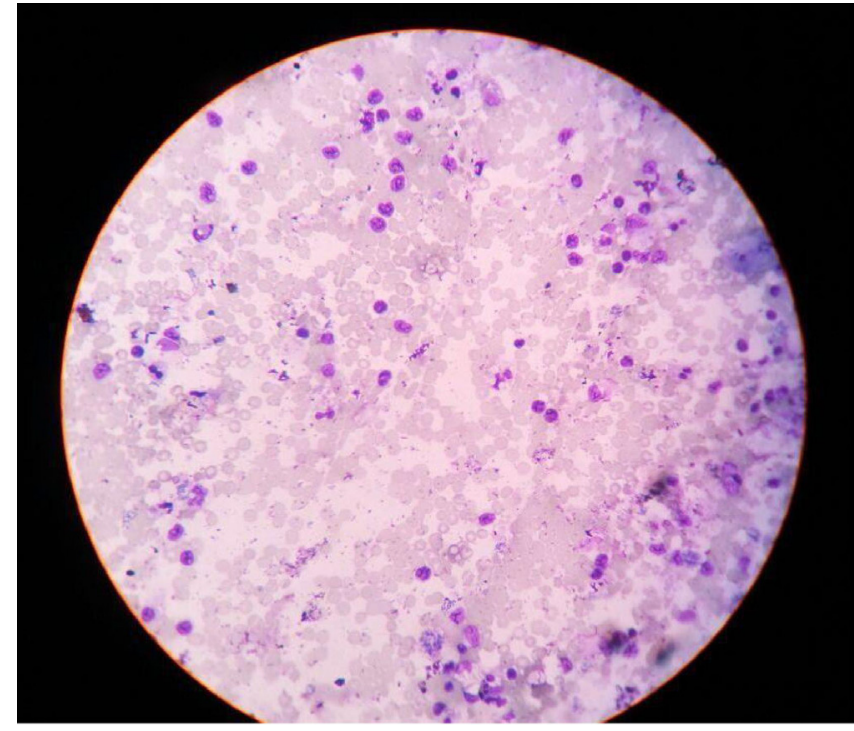

Figure 3. microscopic picture of aqueous tap showing predominantly polymorphonuclear cells.

x-ray sacroiliac joint showed no abnormality. The patient was started on 2hourly topical steroids and oral prednisolone $1 \mathrm{mg} /$ $\mathrm{kg} /$ day for a week with gradual tapering every week, along with dilators. Vision after 2 weeks: OD- 6/6, OS- 6/18(p) improving to $6 / 6(p)$ with pinhole. The anterior chamber was quiet. IOP was normal. Pupillary membranes and vitritis were resolved.

\section{Conclusion}

The clinical situation grossly improved in our case suggesting the importance of meticulous history taking as a whole besides a good ophthalmological evaluation of affected and normal eye keeping all the differentials in mind as uveitis is associated with many systemic diseases.

\section{References}

1. Gallagher MJ, Yilmaz T, Cervantes-Castaneda RA, et al. The characteristic features of optical coherence tomography in posterior uveitis. Br J Ophthalmol. 2007; 91: 1680-85.

2. Menezo V, Bond SK, Towler HM, et al. Cytokine gene polymorphisms involved in chronicity and complications of anterior uveitis. Cytokine. 2006; 35: 200-6.

3. Smith RE, Nozik RA. Uveitis: Goals of Uveitis Management; A clinical approach to diagnosis and management Ed. 2, Williams and Wilkins: Baltimore. 1986; 5: 23-4.

4. Adam B. Raff Cellulitis. JAMA. 2016; 316(3):325-37.

\section{*Correspondence to:}

Shweta Dhiman

Department of Ophthalmology

Delhi

India

Tel: +919711423959

E-mail: sdmbbs@yahoo.com 